\title{
Outcomes after surgical treatment of meningioma-associated proptosis
}

\author{
Christian A. Bowers, MD, ${ }^{1}$ Mohammed Sorour, MBBS, ${ }^{1}$ \\ Bhupendra C. Patel, MD, FRCS, FRCOphth, ${ }^{2}$ and William T. Couldwell, MD, PhD'
}

Departments of ${ }^{1}$ Neurosurgery and ${ }^{2}$ Ophthalmology, University of Utah, Salt Lake City, Utah

\begin{abstract}
OBJECTIVE Meningioma-associated proptosis (MAP) can be cosmetically and functionally debilitating for patients with sphenoorbital and other skull base meningiomas, and there is limited information on the quantitative improvement in proptosis after surgery. Because less extensive removals of tumor involving the orbit fail to reduce proptosis, the senior author has adopted an aggressive surgical approach to the removal of tumor involving the periorbita and orbit. The authors of this study retrospectively reviewed outcomes of this surgical approach.
\end{abstract}

METHODS All surgeries for MAP performed by a single surgeon between January 1, 2002, and May 1, 2015, were reviewed. Age, sex, visual symptoms, number and types of surgical treatments, cavernous sinus involvement, complications, duration of follow-up, residual tumor, use of adjuvant radiation therapy, and extent of proptosis resolution as measured by the exophthalmos index (EI) pre- and postoperatively and at the final follow-up were recorded.

RESULTS Thirty-three patients (24 female [73\%]) with an average age of 51.6 years were treated for MAP. Of the 22 patients with additional visual symptoms (for example, loss of visual acuity, field cut, or diplopia), 15 had improved vision and 7 had stable vision. No patients had worse proptosis after treatment. The average preoperative El was 1.39, the average immediate postoperative El was 1.23, and the average final El at the most recent follow-up was 1.13. Thus, average overall El improvement was 0.26 , but the average immediate El reduction was 0.16 , demonstrating that proptosis progressively improved during the postoperative period. Residual cavernous sinus involvement was present in 17 of 18 patients who had had preoperative cavernous sinus meningioma involvement. Only 2 patients in the series had recurrent tumor at the orbital region, and their proptosis improved again after reoperation. One case of delayed vasospasm and 2 cases of postoperative trigeminal numbness (V2) were recorded. The average follow-up was 4.5 years (53.8 months).

CONCLUSIONS In this series, all patients experienced proptosis improvement and none had worse visual symptoms at the final follow-up, although proptosis resolution occurred over time. Only 2 patients had tumor recurrence at the orbit that required surgery. Surgical complications were uncommon. Study results suggest that aggressive resection of MAP is well tolerated and offers superior proptosis elimination with infrequent recurrence at the orbit. Importantly, no cases of enophthalmos were noted despite the lack of formal reconstruction of the orbit.

http://thejns.org/doi/abs/10.3171/2015.9.JNS15761

KEY WORDS proptosis; meningioma; skull base; vision; oncology

$\mathrm{P}$ ROPTOSIS is typically the presenting sign in patients with sphenoorbital meningiomas (SOMs), although visual deterioration is also frequently observed.11,12 Meningioma-associated proptosis (MAP) can be cosmetically and functionally debilitating for patients with SOM and other skull base meningiomas. Meningiomas make up 95\% of benign brain tumors, but $15 \%-19 \%$ of meningiomas involve the greater sphenoid wing and are also known as "orbitosphenoid meningioma," "meningioma en plaque of the sphenoid wing," and "sphenoid wing meningioma with osseous involvement." These SOMs are uncommon, accounting for just $0.2 \%-9.0 \%$ of all meningiomas. ${ }^{5,12}$
The surgical management of SOM has undergone a significant evolution since the original surgical paper from more than 60 years ago recommended that "these patients as a rule should not be operated on and surgical measures should only be considered as a last resort." 2 This conservative philosophy was partially based on the high surgical mortality rate of $10 \%-15 \%$ at the time. ${ }^{2}$ Although subsequent articles showed that SOMs could be resected with low morbidity and mortality, controversy remains regarding the optimal surgical approach, the extent of resection that should be pursued, the need for periorbital opening and/or stripping, the need for orbital roof and/or wall

ABBREVIATIONS EI = exophthalmos index; MAP = meningioma-associated proptosis; $\mathrm{SOM}=$ sphenoorbital meningioma .

SUBMITTED April 3, 2015. ACCEPTED September 2, 2015.

INCLUDE WHEN CITING Published online January 22, 2016; DOI: 10.3171/2015.9.JNS15761. 
reconstruction, and the type of or the need for duraplasty. ${ }^{59-12}$ Only a few studies have quantitatively examined postoperative proptosis outcomes..$^{5,10-12}$

The surgical procedure performed by the senior author (W.T.C.) has undergone significant evolution over the past 2 decades to achieve more aggressive resection. This is largely because of the unsatisfactory results in reducing proptosis with the less extensive surgery performed early in his career. Here we describe this surgical technique and evaluate our surgical results. We emphasize proptosis outcomes, as measured quantitatively by the exophthalmos index (EI), to assess whether our surgical techniques permit aggressive proptosis reduction with low rates of morbidity and mortality. ${ }^{12}$

\section{Methods}

We performed a retrospective chart review to identify all patients who had undergone surgical treatment for MAP performed by the senior author between January 1, 2002, and May 1, 2015. Patients whose original films were not available for an initial EI measurement, whose EI was $\leq 1.1$, or who had another pathology causing proptosis, such as fibrous dysplasia, were excluded. We recorded age, sex, type and duration of visual symptoms (if present), number and types of surgical treatments, cavernous sinus involvement, complications, length of follow-up, presence of residual tumor, use of adjuvant radiation therapy, and extent of proptosis resolution. Proptosis was quantitatively compared by measuring the EI demonstrated on neuroimaging preoperatively, immediately postoperatively during the hospital stay, and at the most recent follow-up. The exophthalmos index was described by Scarone et al. ${ }^{12}$ and is determined by drawing a horizontal line between the tips of the frontal processes of the zygoma and then calculating the ratio of the distances from that line to the anterior aspect of the globe for each eye.

All chart reviews and data acquisition were in compliance with regulations determined by the University of Utah Institutional Review Board and with the Health Insurance Portability and Accountability Act (HIPAA) of 1996.

\section{Surgical Technique}

Preoperative MRI, with and without intravenous contrast, and CT images are carefully studied, with focus on the extent of tumor. The amount of bone hyperostosis and soft tissue contribution to the proptosis determines the amount of bone drilling required to achieve adequate decompression of the orbit.

The patient is placed supine on the operating table. The head is rotated approximately $30^{\circ}-35^{\circ}$ to the contralateral side and placed in a Mayfield head holder. Total intravenous general anesthesia is induced. In approaching sphenoorbital tumors, a standard frontotemporal craniotomy is performed. In many cases, direct involvement of the lateral sphenoid region is evident (Video 1).

VIDEO 1. Case 24. Operation demonstrating right SOM with proptosis. Copyright Department of Neurosurgery, University of Utah. Published with permission. Click here to view.

A frontotemporal bone flap is fashioned and removed. Hyperostotic bone is resected with a high-speed cutting bur.
The lateral and superior orbital wall is then drilled to the periorbita. Bone hyperostosis can be severe, necessitating extensive drilling. The bone flap is planned to incorporate all involved bone of the lateral sphenoid wing. If the hyperostotic bone involves the bone flap, this portion of the flap is resected prior to reimplantation of the bone flap. The involved lateral sphenoid bone is then drilled with a highspeed cutting bur until the periorbita and dura mater are in proximity. Care is taken to avoid tearing the periorbita at this stage.

The lateral orbital wall is removed entirely, the superior orbital fissure and foramen rotundum are identified, and all bone is removed around them. If the middle fossa bone appears hyperostotic on the preoperative CT scan, all of the involved bone is removed in its entirety. The goal of resection is to remove all involved bone, as it is assumed to contain viable meningioma. In almost all cases, bone hyperostosis involves the orbital walls and does not involve the orbital rim. In these cases, the orbital rim is left intact if it is not involved with hyperostotic changes, and all of the bone of the lateral and superior walls just posterior to the rim is removed (Fig. 1).

After the entire superior and lateral wall is removed (Fig. 1), the optic canal is unroofed and the clinoid process is removed (described in detail elsewhere ${ }^{3}$ ). If tumor involves the periorbita, then this is removed as well. The surgical technique entails careful microsurgical dissection of tumor-involved periorbita from the underlying orbital fat and extraocular muscles (Video 1). A plane is developed and followed to the interface with orbital contents. If extraocular muscles are involved with the tumor, the surgeon must decide whether to resect them. In younger patients, we have elected to remove tumor completely for better tumor control because tumor progression in this location will invariably produce extraocular motion limitation.

The orbit is not formally reconstructed after the tumor and periorbita are removed. A muscle plug (obtained from the exposed temporalis muscle) is used to close off any defect into the paranasal sinuses that have been entered with the resection (usually frontal, ethmoid, and sphenoid sinuses). A piece of autologous fascia and fat is used to separate orbital contents from the surrounding frontal lobe and temporal lobe dura (Video 1).

\section{Results}

\section{Patient Demographics}

During the study period, 33 patients underwent craniotomy for MAP treatment (Table 1). The average age was 51.6 years and $73 \%$ of the patients were female (24 of 33). Twenty-two patients had other preoperative visual symptoms in addition to proptosis: Approximately onethird of the patients (12 [36.4\%] of 33) had a visual field defect, and over half (17 [51.5\%] of 33) had a loss of visual acuity. Periorbital pain was also present in almost half of the patients (14 [42.4\%] 33), and diplopia was present in $36.4 \%$ of patients (12 of of 33). Six (18.2\%) patients had undergone subtotal resection at an outside institution prior to presenting to us with a recurrence. The average preoperative duration of visual symptoms other than proptosis, when present, was 15.2 months (range $0-130$ months). 

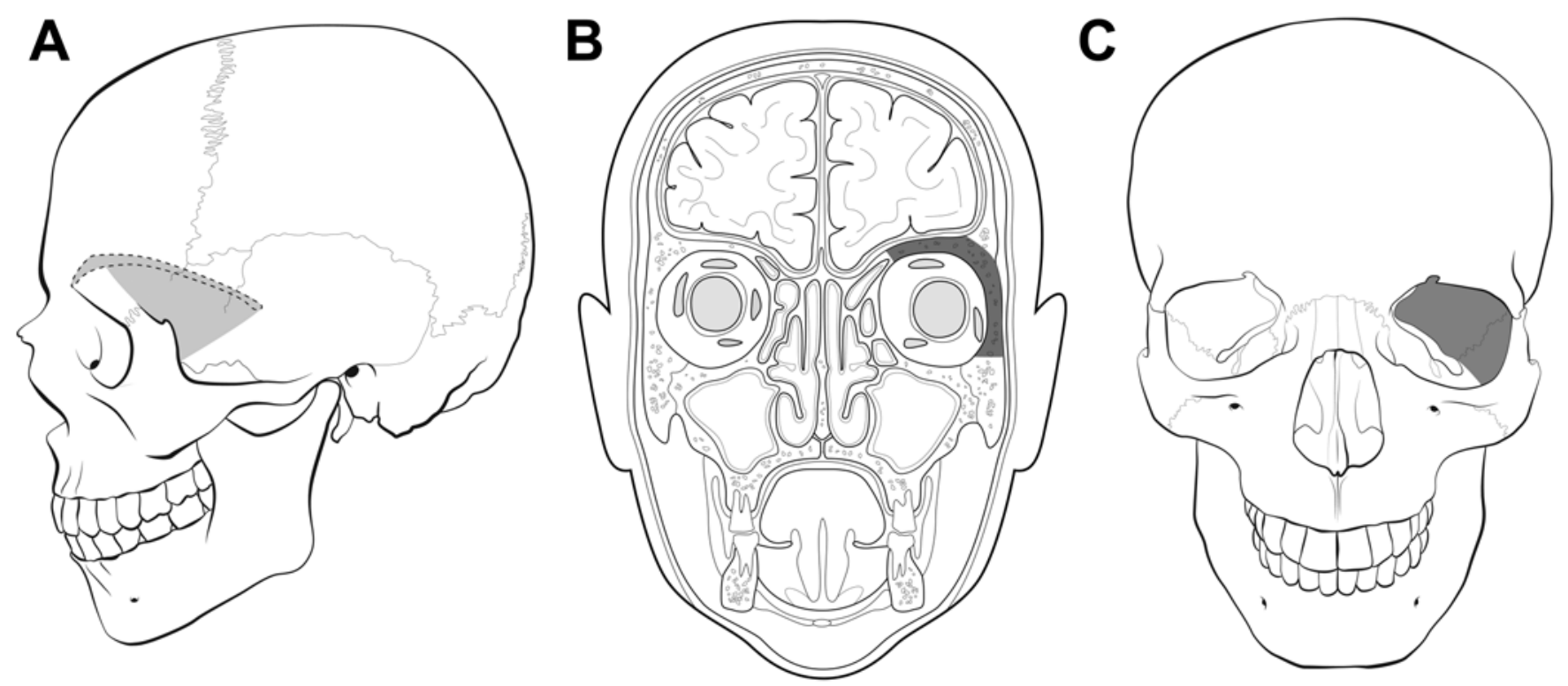

FIG. 1. Shading indicates the area of bone removal from an external lateral view (A), internal coronal view (B), and external coronal view (C). Shaded areas include the lateral wall, superior wall, optic canal, and clinoid process. We preserved the orbital rim. Copyright Department of Neurosurgery, University of Utah. Published with permission.

\section{Surgical Outcomes}

The cavernous sinus was involved in the majority of patients (18 [54.5\%] of 33), and residual cavernous sinus tumor was present in 17 of these patients after surgery. All but 1 of these latter 17 patients received radiation therapy via stereotactic radiosurgery to prevent tumor progression. Of the 22 patients with additional visual symptoms preoperatively, 15 had improved vision and 7 had stable vision. Periorbital stripping was performed whenever there was periorbital tumor involvement (27 [81.8\%] of 33 patients). The stripping was done to all of the periorbita that was infiltrated by tumor, posteriorly to the limit of the annulus of Zinn. Surgical complications were rare: there was 1 case of delayed vasospasm, and 2 patients had persistent trigeminal (V2) numbness. Two patients had complications after radiation therapy to the cavernous sinus: 1 with abducens neuropathy and 1 with pituitary insufficiency. The revision surgeries performed in patients who had undergone previous surgical treatment at an outside institution were more technically challenging, but there was no association with increased complication or worse proptosis outcomes. Ten patients (30.3\%) needed an additional surgery to remove their tumor. Five of these patients had planned staged surgeries for larger tumors that extended into the posterior fossa or into the sphenoidal or ethmoid sinus regions, necessitating different surgical approaches for gross-total resection. Three other patients had delayed intraconal surgery for recurrent meningioma from residual tumor involving the intraocular muscles that had been deliberately left behind to preserve function. At the second surgery in these cases, tumor-involved muscle was removed. The other 2 patients had unanticipated recurrent tumor at the sphenoorbital region, and their proptosis improved again after reoperation. The average follow-up was nearly 4.5 years (53.8 months).

\section{Exophthalmos Index}

The average preoperative EI was 1.39, and the average final EI at the most recent follow-up was 1.13, yielding an average EI improvement of 0.26 (Fig. 2). The average immediate postoperative EI for all patients was only 1.23 , for an average initial EI reduction of 0.16 , demonstrating that proptosis progressively improved during the postoperative period. Importantly, no patients experienced enophthalmos or worse proptosis on the final follow-up despite aggressive resection. No patients experienced worsened vision after treatment.

\section{Illustrative Cases}

\section{Case 24}

\section{History and Examination}

A 66-year-old woman presented to our hospital having experienced difficulty swallowing water and progressive neurological deterioration, with rapid deterioration in the days prior to admission. Seventeen years earlier, an SOM had been radiographically diagnosed by an outside hospital after she had presented with proptosis of the right eye; however, she avoided medical treatment because she was told that her lesion was inoperable. On presentation to our facility, she had extreme proptosis with an EI of 2.41 (Fig. 3A), and preoperative MRI and CT showed an enormous right-sided SOM with chronic uncal herniation, brainstem compression, and $1.4 \mathrm{~cm}$ of midline shift with associated hydrocephalus (Fig. 3B-E). Preoperative vision was 20/400 in the right eye. She also had significant right hemiparesis and was drowsy, although she was oriented to self, location, and time.

\section{Operation}

The patient underwent a right frontotemporal craniot- 


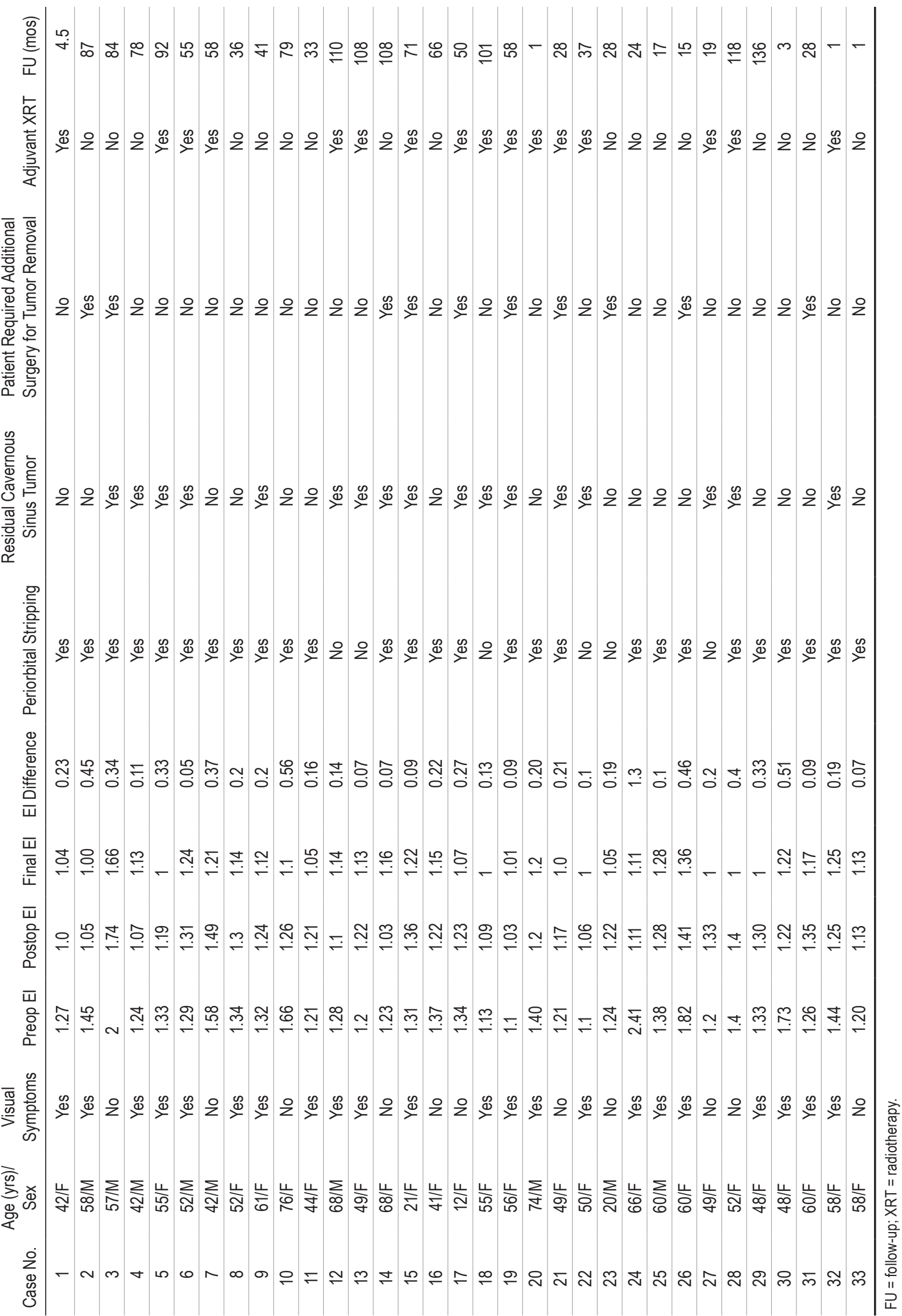




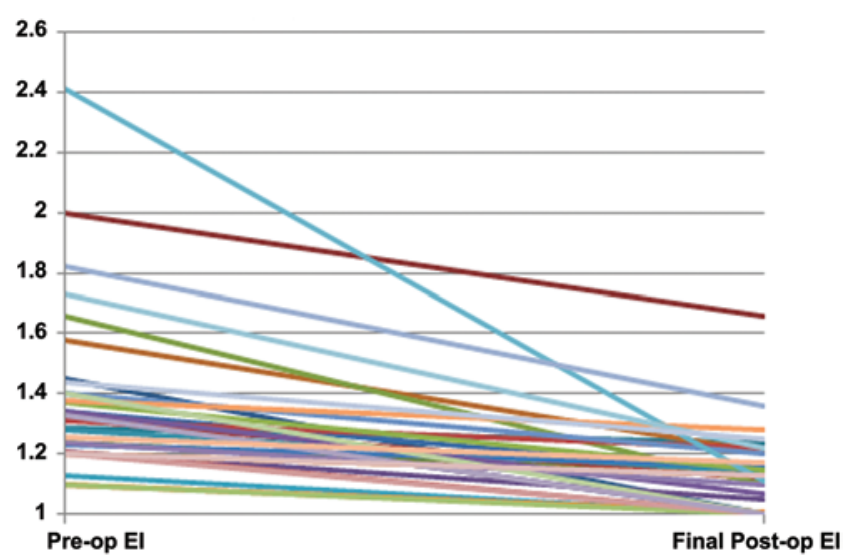

FIG. 2. Graph demonstrating El outcomes with preoperative El (left) and final postoperative El (right). Figure is available in color online only.

omy for orbital decompression and resection of the SOM (Video 1). A cutting bur was used to create several bur holes circumferentially at the interface between abnormal tumor bone and normal frontotemporal bone on the periphery of the lesion. A footplate was then used to connect the multiple bur holes. Attention was then turned to drilling down the extremely thick hyperostotic abnormal meningiomainvolved bone. The involved bone in the frontal, temporal, orbital, and sphenoidal regions was carefully drilled away using a high-speed cutting bur. Abnormal tumor-involved bone in the temporal fossa was removed with drilling as well. The dura was then opened, and the intradural tumor component was removed along with the attached dura. The orbital portion of tumor was easily removed with dissection, and the involved periorbita was then removed. Finally, an AlloDerm dural graft was used to cover the large area of dura removed with the tumor resection.

\section{Postoperative Course}

The patient recovered for a week in the intensive care unit and was discharged to inpatient rehabilitation after another week on the general neurosurgical ward. Immediate postoperative head CT showed a significant reduction in proptosis with an immediate postoperative EI of 1.11 (EI reduction of 1.3; Fig. 3F). She required a 2-month inpatient rehabilitation stay to address her baseline hemiparesis and dysphagia, and a custom cranioplasty was implanted during that stay. At discharge, she required only minor assistance from family and has had no further proptosis recurrence. She has experienced a dramatic cosmetic improvement as well (Fig. 3G).

\section{Case 7}

History and Examination

A 33-year-old man diagnosed with SOM was treated
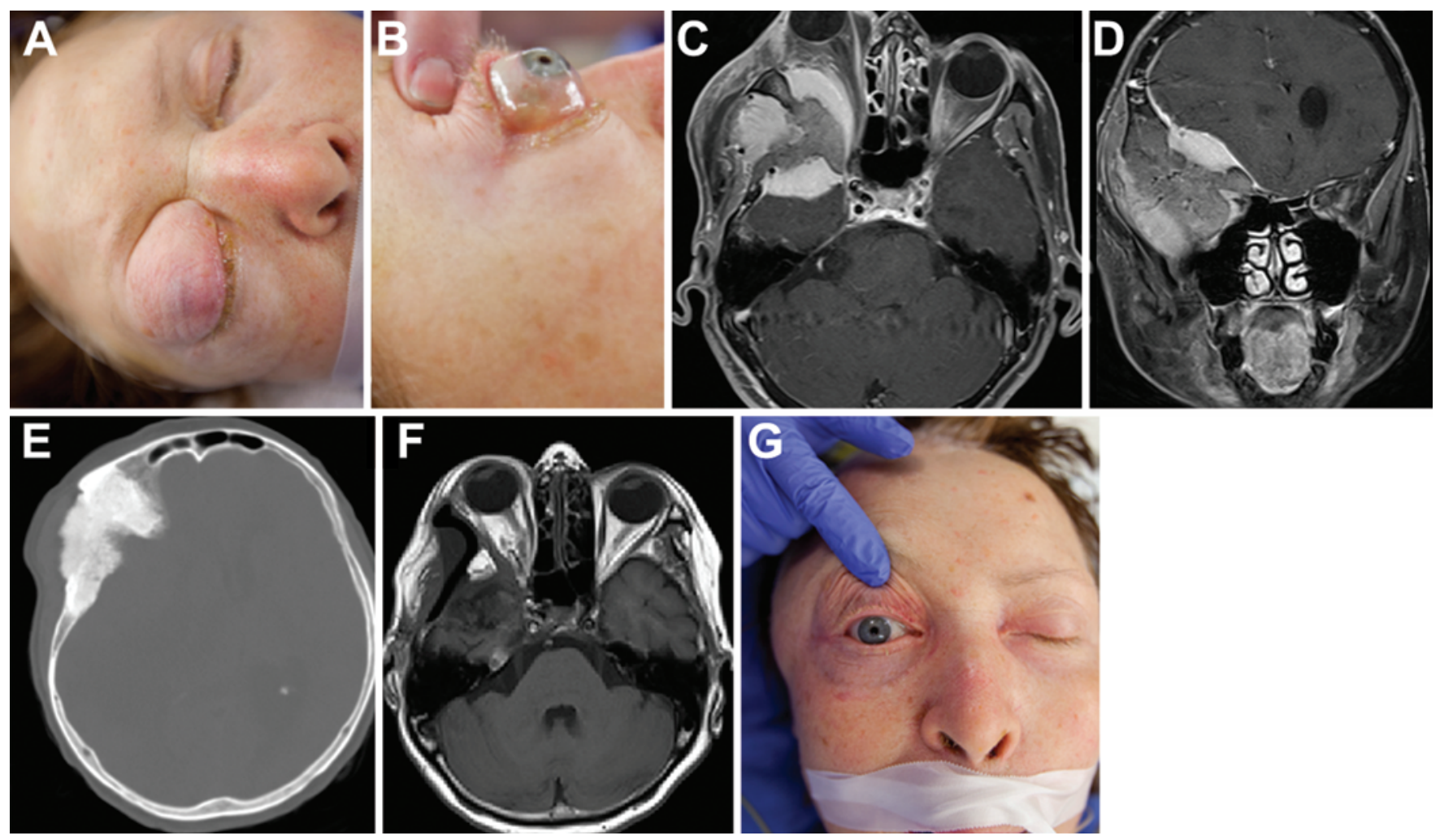

FIG. 3. Case 24. A and B: Preoperative photographs demonstrating significant right eye proptosis. C: Preoperative axial T2-weighted MR image demonstrating significant proptosis and the extent of optic nerve compression $(E I=2.41)$. D: Coronal T2weighted MR image showing the significant width of tumor infiltration in the sphenoorbital region. E: Preoperative axial CT scan demonstrating the reactive hyperostosis and extent of bony involvement with this tumor. F: Postoperative axial T1-weighted MR image demonstrating excellent proptosis resolution $(E I=1.11)$. G: Postoperative photograph demonstrating good resolution of the right eye proptosis. Figure is available in color online only. 
at an outside facility with subtotal resection that did not resolve his proptosis. Eight years later, another subtotal resection also failed to reduce his proptosis significantly, so he underwent radiation therapy 6 months later. Six months after radiation, at the age of 42 years, he presented to our clinic with persistent proptosis, left orbital congestion that was exacerbated by lowering his head, and undesirable cosmesis due to the significant exophthalmos. He had no visual symptoms and was neurologically intact, but his EI was 1.58 (Fig. 4A and B).

\section{Operation and Postoperative Course}

The patient underwent a repeat left frontotemporal craniotomy. The superior and lateral orbital walls were removed, and an anterior clinoidectomy was done as well. The extradural dissection and drilling of the abnormal bone continued down to the inferotemporal fossa. The 3 branches of the trigeminal nerve were skeletonized at the base of the skull, and all abnormal bone was completely removed. The only dura removed was that overlying the orbit, and a dural graft was used in closing this defect. The orbital wall was not reconstructed.

The patient's immediate postoperative EI was 1.49, and his most recent follow-up EI was 1.21, for a final EI reduction of 0.37 from his original EI of 1.58 (Fig. 4C). He was discharged home neurologically intact after a few days in the hospital.

\section{Discussion}

This retrospective single-institution study demonstrates the extent of proptosis reduction that can be achieved with aggressive surgical treatment of MAP with a low incidence of morbidity and mortality. The average 0.26 reduction in the mean preoperative EI from 1.39 to 1.13 reveals significant proptosis improvement by bringing the final postoperative EI to near normal (EI of 1.00). None of the patients had worse postoperative proptosis at the final follow-up as compared with their preoperative EI. Furthermore, none of the 22 patients with visual symptoms had worse vision postoperatively, and $68.2 \%$ of them (15 of 22) had significant visual improvement. No cases of enophthalmos were noted.

\section{Proptosis Resolution and Surgical Management of the Periorbita}

In the past several years, several publications have addressed proptosis outcomes with SOM as well as the surgical strategies for dealing with SOM-involved periorbita. ${ }^{5,-12}$ Surgical techniques along a spectrum have also been described, ranging from leaving the periorbita alone ${ }^{9}$ to "periorbital stripping" and partial resection ${ }^{10}$ to complete periorbital incision and resection. ${ }^{12}$ The technique of not disrupting the periorbita is implemented primarily to prevent enophthalmos development. However, another proposed rationale is that tumor recurrence predominantly comes from the cavernous residual tumor and not from the residual meningioma-involved periorbita, so there is no need to be surgically aggressive with the periorbitainvolved tumor. ${ }^{9}$ There is also a widely cited belief that periorbital opening may necessitate orbital reconstruction to prevent cosmetic deformity or impaired functionality with the development of enophthalmos. . $^{1,4,6,8,10}$

Because earlier reports lacked a standard quantitative method of exophthalmos measurement for comparison, it is difficult to directly compare proptosis outcomes associated with the various techniques. ${ }^{12}$ Quantitative measurements are important because of the wide range of ocular globe variability and because large SOMs disrupt visualization of the orbital apex, which impedes traditional clinical exophthalmos measurements. ${ }^{12}$ Proptosis outcomes have traditionally been reported qualitatively as proptosis "improvement," which was achieved in 70\%-96\% of patients in these surgical series; ${ }^{9,12}$ however, patients can have a high percentage of reported proptosis "improvement" while still suffering from significant proptosis. Residual proptosis rates after SOM surgery span a wide range from $23 \%$ to $76 \%$, and the length of follow-up significantly affects proptosis outcomes since recurrence rates can be as high as 50\% in some series. . $^{5,-12}$

Proptosis resolution was excellent in our series: the final EI of 1.13 yielded an average EI improvement of 0.26 and approached a normal EI of 1.0. Furthermore, none of our patients experienced enophthalmos or worse proptosis despite aggressive periorbital incision and/or resection and orbital wall removal. The surgical challenge in this disease is reflected in the 6 patients who had undergone a remote
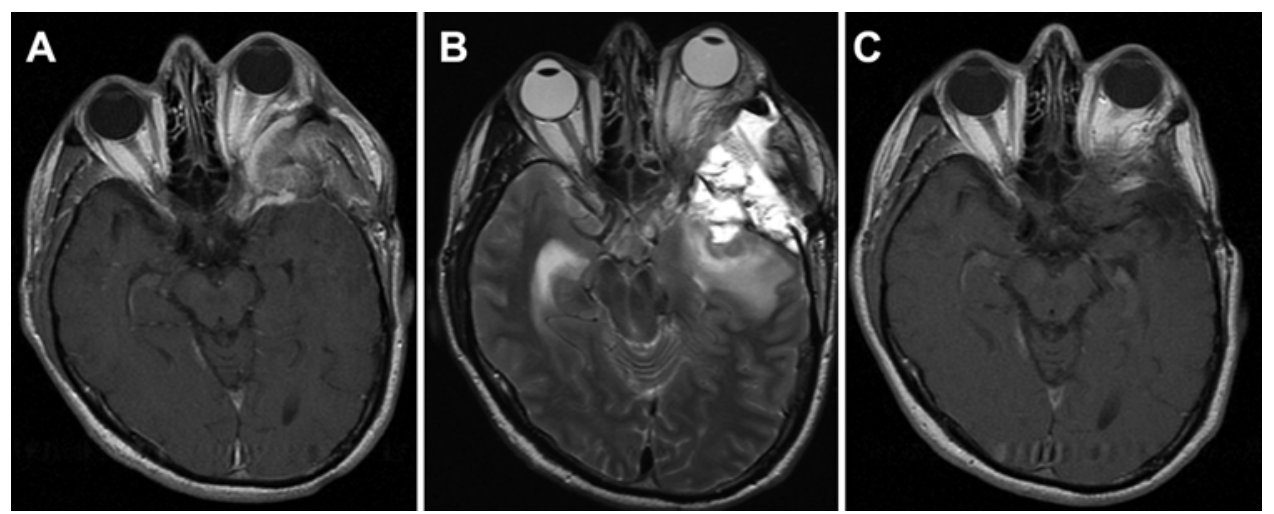

FIG. 4. Case 7. A: Preoperative T1-weighted MR image with gadolinium enhancement. B: Preoperative T2-weighted MR image. C: Final postoperative T1-weighted MR image with gadolinium enhancement demonstrating the significant proptosis reduction: from El 1.58 to 1.21 . 
surgery at an outside institution. We believe the key surgical technique of periorbita incision and resection from the orbital fat (when there is periorbita-SOM involvement) to achieve maximal proptosis elimination is responsible for the good proptosis outcomes reported in this series. This periorbital stripping technique was performed whenever there was periorbital invasion (27 [81.8\%] of 33 of our patients).

\section{Orbital Reconstruction}

The indications for and techniques of orbital reconstruction as defined in previous reports have been inconsistent. ${ }^{4,510-12}$ Traditionally, orbital wall reconstruction was thought to be necessary to avoid pulsatile enophthalmos whenever more than one orbital wall was removed. ${ }^{10,11}$ Two large series ${ }^{4,7}$ were subsequently published to show that orbital wall reconstruction was not necessary even when complete orbital roof resections were coupled with lateral or medial orbital wall resections; $;, 7,10,11$ however, these conclusions were based on the belief that an intact periorbita prevented the development of enophthalmos.,10,11 In the present study enophthalmos was not encountered postoperatively in patients who had presented with proptosis, despite a lack of formal orbital reconstruction. The reason patients with MAP do not experience enophthalmos or significant oscillopsia is not established; however, in our experience, the periorbital tissues show a "woody" or "firm rubbery" consistency, which, we believe, makes the elimination of proptosis difficult but may also account for the lack of significant pulsatile enophthalmos following the extensive decompression. We only reconstruct the orbit on the very rare occasion when the orbital rim must be removed. Although we almost never have to remove the orbital rim, different materials have been used for orbital rim reconstruction, including calvarial split-thickness grafts, methylmethacrylate, and other synthetic grafts.

\section{Study Limitations}

The principal limitation of this study is that it is a retrospective cohort study from a single surgeon and institution. Therefore, we recommend our results foster further prospective study of SOM surgical techniques and quantitative proptosis outcomes by other surgeons.

\section{Conclusions}

Aggressive resection of MAP resulted in significant proptosis improvement, with a mean preoperative EI of 1.39 and a final mean follow-up EI of 1.13. A critical microsurgical technique to achieve optimal proptosis reduction when there is periorbital tumor invasion is the careful dissection of tumor-involved periorbita from the underlying orbital fat and extraocular muscles. All patients in this series experienced proptosis improvement, and none had worsened visual symptoms or proptosis on follow-up. Importantly, no cases of enophthalmos were noted. We found that proptosis resolution takes time (several months), as the immediate postoperative proptosis reduction was much closer to the preoperative EI than the final EI at the most recent follow-up.

\section{References}

1. Adkins WY Jr: Maxillectomy with preservation of orbital function. Surg Forum 27:548-550, 1976

2. Castellano F, Guidetti B, Olivecrona H: Pterional meningiomas en plaque. J Neurosurg 9:188-196, 1952

3. Couldwell WT, Kan P, Liu JK, Apfelbaum RI: Decompression of cavernous sinus meningioma for preservation and improvement of cranial nerve function. Technical note. J Neurosurg 105:148-152, 2006

4. DeMonte F, Tabrizi P, Culpepper SA, Suki D, Soparkar CN, Patrinely JR: Ophthalmological outcome after orbital entry during anterior and anterolateral skull base surgery. $\mathbf{J}$ Neurosurg 97:851-856, 2002

5. Heufelder MJ, Sterker I, Trantakis C, Schneider JP, Meixensberger J, Hemprich A, et al: Reconstructive and ophthalmologic outcomes following resection of sphenoorbital meningiomas. Ophthal Plast Reconstr Surg 25:223226, 2009

6. Larson DL, Christ JE, Jesse RH: Preservation of the orbital contents in cancer of the maxillary sinus. Arch Otolaryngol 108:370-372, 1982

7. Maroon JC, Kennerdell JS, Vidovich DV, Abla A, Sternau L: Recurrent spheno-orbital meningioma. J Neurosurg 80:202208, 1994

8. McCary WS, Levine PA: Management of the eye in the treatment of sinonasal cancers. Otolaryngol Clin North Am 28:1231-1238, 1995

9. Oya S, Sade B, Lee JH: Sphenoorbital meningioma: surgical technique and outcome. J Neurosurg 114:1241-1249, 2011

10. Ringel F, Cedzich C, Schramm J: Microsurgical technique and results of a series of 63 spheno-orbital meningiomas. Neurosurgery 60 (4 Suppl 2):214-222, 2007

11. Saeed P, van Furth WR, Tanck M, Freling N, van der Sprenkel JW, Stalpers LJ, et al: Surgical treatment of sphenoorbital meningiomas. Br J Ophthalmol 95:996-1000, 2011

12. Scarone P, Leclerq D, Héran F, Robert G: Long-term results with exophthalmos in a surgical series of 30 sphenoorbital meningiomas. Clinical article. J Neurosurg 111:1069-1077, 2009

\section{Disclosures}

The authors report no conflict of interest concerning the materials or methods used in this study or the findings specified in this paper.

\section{Author Contributions}

Conception and design: Couldwell. Acquisition of data: Bowers. Analysis and interpretation of data: Bowers, Sorour. Drafting the article: Bowers. Critically revising the article: Couldwell, Bowers, Patel. Reviewed submitted version of manuscript: all authors. Approved the final version of the manuscript on behalf of all authors: Couldwell.

\section{Supplemental Information \\ Videos}

Video 1. https://vimeo.com/143261774.

\section{Previous Presentations}

This work was presented as an oral talk at the 2015 AANS meeting held in Washington, DC, on May 2-6, 2015.

\section{Correspondence}

William T. Couldwell, Department of Neurosurgery, Clinical Neurosciences Center, University of Utah, 175 N. Medical Dr. E, Salt Lake City, UT 84132. email: neuropub@hsc.utah.edu. 